\title{
Stool and ruminal microbiome components associated with methane emission and feed efficiency in Nelore beef cattle.
}

Bruno G.N. Andrade

Embrapa Pecuaria Sudeste

Haithem Afli

Cork Institute of Technology

Flavia A. Bressani

Embrapa Pecuaria Sudeste

Rafael R. C. Cuadrat

Dife

Priscila S. N. de Oliveira

Embrapa Pecuaria Sudeste

\section{Gerson B. Mourão}

Universidade de Sao Paulo Escola Superior de Agricultura Luiz de Queiroz

\section{Luiz L. Coutinho}

Universidade de Sao Paulo Escola Superior de Agricultura Luiz de Queiroz

James M. Reecy

lowa State University

James E. Koltes

lowa State University

Marcela Maria de Souza

lowa State University

Adhemar Zerlotini Neto

Embrapa Informatica Agropecuaria

Sérgio Raposo de Medeiros

Embrapa Pecuaria Sudeste

Alexandre Berndt

Embrapa Pecuaria Sudeste

Julio C. P. Palhares

Empresa Brasileira de Pesquisa Agropecuaria

Luciana C. A. Regitano ( $\square$ luciana.regitano@embrapa.br)

Embrapa Pecuaria Sudeste https://orcid.org/0000-0001-9242-8351 
Research

Keywords: Association, archaea, bacteria, Bos Indicus, Biomarkers, Feed efficiency, methane emission

Posted Date: November 16th, 2021

DOI: https://doi.org/10.21203/rs.3.rs-74103/v2

License: (c) (i) This work is licensed under a Creative Commons Attribution 4.0 International License. Read Full License

Version of Record: A version of this preprint was published at Frontiers in Genetics on May 17th, 2022. See the published version at https://doi.org/10.3389/fgene.2022.812828. 


\section{Abstract}

Background: The impact of extreme changes in weather patterns in the economy and human welfare are some of the biggest challenges that our civilization is facing. From the anthropogenic activities that contribute to climate change, reducing the impact of farming activities is a priority, since it is responsible for up to $18 \%$ of greenhouse gases linked to such activities. To this end, we tested if the ruminal and fecal microbiome components of 52 Brazilian Nelore bulls, belonging to two treatment groups based on the feed intervention, conventional and by-products based diet, could be used in the future as biomarkers for methane emission and feed efficiency in bovine.

Results: We identified a total of 5,693 Amplicon Sequence Variants (ASVs) in the Nelore bulls microbiomes. Differential abundance (DA) analysis with the ANCOM approach identified 30 bacterial and 15 archaea ASVs as DA among treatment groups. Association analysis using Maaslin2 and Mixed Linear Models indicated that bacterial ASVs are linked to the residual methane emission $\left(\mathrm{RCH}_{4}\right)$ and Residual Feed Intake (RFI) phenotypes, contributing to the host's phenotypic variation, suggesting their potential as targets for interventions and/or biomarkers.

Conclusion: Feed composition induced significant differences in abundance and richness of ruminal and fecal microbial populations. The diet based on industrial byproducts applied to our treatment groups influenced the microbiome diversity of bacteria and archaea, but not of protozoa. Different ASVs were associated with $\mathrm{RCH}_{4}$ emission and RFI in both ruminal and fecal microbiomes. While ruminal ASVs are expected to directly influence $\mathrm{RCH}_{4}$ emission and $\mathrm{RFI}$, the relation of fecal taxa, such as Alistipes and Rikenellaceae (gut group RC9), with these traits might also be associated with host health due to their link to anti-inflammatory compounds, and these have the potential to be used as accessible biomarkers for these complex phenotypes.

\section{Background}

The Anthropocene Epoch is marked by the continuous degradation of the biosphere promoted by human activity, which ultimately culminated in the ongoing climate and environmental crisis (Williams et al., 2015; Cook et al., 2016). One of the current challenges is to mitigate its effects and sustain an evergrowing human population through the development of methods for efficient food production. Cattle farming, a valuable source of animal protein, is responsible alone for a significant environmental impact due to the occupation and degradation of land for pasture, contamination of water sources by cattle manure (Sahoo et al., 2016), and the emission of methane $\left(\mathrm{CH}_{4}\right)$ produced by enteric fermentation, a greenhouse gas 28 times stronger than $\mathrm{CO}_{2}$ (Pachauri et al., 2015).

Key phenotypes for the reduction of meat industry's environmental and economical burden, such as feed efficiency, and methane emission, are linked to interactions between the host, and its associated microbial communities, also known as the microbiota (Guan et al., 2008; Chang et al., 2019). Although studies targeting these phenotypes have been published over the years (Mudadu et al., 2016; Pszczola et 
al., 2018; Lassen and Difford, 2020), the microbiota only started to be considered as an important subject to studies aiming to increase efficiency, reduce costs and the environmental impact of cattle farming in the past few years (Shi et al., 2014; Noel et al., 2019).

These microorganisms can shape their host biology through beneficial interactions, and thereby influence health, development, and immune system modulation (Flint et al., 2007; Belkaid and Hand, 2014). However, most of these microorganisms are elusive and utterly unknown to science due to inherent difficulties related to cultivation procedures (Solden et al., 2016). Nowadays, it is possible to access microorganisms' genomic material directly, the so-called microbiome, and investigate their identity, distribution, relatedness, and functionality using approaches from the meta'omics field, such as deep sequencing metagenomics, metabarcoding, and metatranscriptomics (Gilbert and Dupont, 2011).

The microbiome structure of the Nelore Brazilian beef cattle has gained the attention of the scientific community in the past years, being subject of different studies (de Oliveira et al., 2013; Lopes et al., 2021), including a previous study by our research group (Andrade et al., 2020). In this study, we investigated the microbiome profiles of two segments of 26 Nelore bulls' gastro-intestinal tract (GIT), and showed that a significant part of the stool archaeal population co-occurred with the rumen archaeal population, suggesting the use of stool as a proxy for the rumen archaeal population.

Herein, we extended that study by the introduction of an additional experimental group under a different diet and compared the microbiome populations from two distant sections of the Nelore gastrointestinal tract (GIT)- rumen and rectal ampulla in order to: (i) identify the impact of the dietary treatment on the microbiome diversity and abundance; (ii) identify associations between microbiome components and phenotypes, such as residual $\mathrm{CH}_{4}$ emission $\left(\mathrm{RCH}_{4}\right)$, and residual feed intake (RFI).

\section{Methods}

\section{Experimental design}

All experimental procedures were conducted in accordance with animal welfare guidelines and were approved by the EMBRAPA Livestock Science Ethics Committee on Animal Experimentation, São Carlos, São Paulo (Protocol No. 09/2016). The experimental population consisted of animals born in 2014, and the experiment was conducted at the feedlot facility of "Embrapa Pecuária Sudeste" and lasted 105 days, which included 15 days for animal adaptation to the feedlot, 30 days for growth, and 60 days for animal finishing. Animals were divided into two groups based on dietary treatment. The first experimental group (conventional group, $n=26$ ), consisted of animals fed with a conventional diet based on corn silage, corn, and soybean meals, rumen protected fat and urea as a concentrate. The second experimental group (byproduct group, $\mathrm{n}=26$ ) had a total replacement of concentrates by the industrial by-products citrus pulp, corn germ, corn germ oil meal, and peanut shell meal. In both treatment groups, animals received mineral supplements, active dry yeast, virginiamycin, and monensin. 
Feedlots were divided based on the dietary treatment and initial weights, with heavyweight and lightweight animals grouped separately. The facility has collective stalls with an automatic feeding system (GrowSafe Systems Ltd., Airdrie, Alberta, Canada), used to collect data regarding live weight and daily food consumption. The animals were then sent for slaughter at 23-24 months of age, in accordance with the Humane Slaughter of Cattle guidelines. All animal data used in this study is available in Supplementary Table 1.

\section{Host DNA extraction and genotyping}

For each animal, $5 \mathrm{ml}$ blood samples were collected, and DNA extractions were performed by a saltingout method. DNA concentration was measured by spectrophotometry, and quality was verified by the $260 / 280$ optical density ratio, followed by inspection of integrity through agarose gel electrophoresis. All animals were genotyped using the GGP Bos indicus 50k. Genotypes were called in the Illumina GenomeStudio software. Samples were filtered according to call rate (95\%), minimum allele frequency (95\%) and monomorphic SNPs. A total of 43,439 SNP were available for further analysis.

\section{Phenotypes}

\section{Residual feed intake}

Individual dry matter intake ( $\mathrm{DMl}, \mathrm{kg} / \mathrm{d}$ ) was obtained by the difference between weight of the diet provided and refusal and average daily gain (ADG, $\mathrm{kg} / \mathrm{d}$ ) was estimated by linear regression of body weight (BW) on days in feedlot. Residual feed intake (RFI, $\mathrm{kg} / \mathrm{d}$ ) was computed as the residuals from regression of DMI on mid-test $B W_{0.75}$ and $A D G$ (Koch et al., 1963). The metabolic body weight (MBW, $\left.\mathrm{kg}\right)$ was obtained with the following equation: $\mathrm{MBW}=\mathrm{BW}^{0.75}$. The contemporary group (CG) was defined as weighing group and slaughter group, which were considered fixed effects by MIXED procedure of SAS statistical program (SAS Institute, Cary, NC, USA, 2011), according to the following equation:

$\mathrm{DMI}_{i}=\beta_{0}+\beta_{1}\left(\mathrm{ADG}_{i}\right)+\beta_{2}\left(\mathrm{MBW}_{i}\right)+\beta_{3}\left(\mathrm{CG}_{i}\right)+\mathrm{RFI}_{i}$

Where $\mathrm{DMl}_{i}$ is the dry matter intake predicted for animal $i, \mathrm{ADG}_{i}$ is the average daily gain of animal $i$; $\mathrm{MBW}_{i}$ is the metabolic body weight of animal $i ; \beta_{0}$ is the regression intercept; $\beta_{1}$ is the partial regression coefficient of $A D G ; \beta_{2}$ is the partial regression coefficient of MBW, $\beta_{3}$ is the partial regression coefficient of $\mathrm{CG}$, and $\mathrm{RFI}_{i}$ is the RFI of animal $i$ (Koch et al., 1963). 


\section{Residual methane emission}

The methane emission was measured during the finishing period in the feedlot using the GreenFeed system (C-lock Inc., Rapid City, SD, USA). The Residual methane emission (RME) was obtained by the difference between methane emission and individual dry matter intake (DMI, kg/d) (Donoghue et al., 2016), where contemporary group (CG), which were considered fixed effects by MIXED procedure of SAS statistical program (SAS Institute, Cary, NC, USA, 2011), according to the following equation:

$M E_{i}=\beta_{0}+\beta_{1}\left(\mathrm{DMI}_{i}\right)+\beta_{2}\left(\mathrm{CG}_{i}\right)+\mathrm{RME}_{i}$

Where $\mathrm{ME}_{i}$ is the methane emission predicted for animal $i ; \mathrm{DMl}_{i}$ is the dry matter intake predicted for animal $i ; \beta_{0}$ is the regression intercept; $\beta_{1}$ is the partial regression coefficient of $\mathrm{DMl} ; \beta_{2}$ is the partial regression coefficient of $\mathrm{CG}$; and $\mathrm{RME}_{i}$ is the residual methane emission proposed by Donoghue et al. (2016) of animal $i$.

\section{Microbiota sample collection and processing}

After the finishing phase, approximately $10 \mathrm{~g}$ of stool was obtained from each animal two weeks before slaughtering, and $50 \mathrm{~mL}$ of rumen content was collected immediately after slaughter. All samples were frozen in liquid nitrogen and permanently stored at $-80^{\circ} \mathrm{C}$ prior to analysis. DNA extraction was performed using the Quick-DNA ${ }^{\mathrm{TM}}$ Fecal/Soil Microbe Miniprep Kit (ZYMO Research Corp., Irvine, CA), following the standard protocol. PCR target amplification for 16S and 18S, PCR conditions, sequencing libraries and DNA sequencing were performed as described in Andrade et al. (2020).

\section{Data retrieval, pre-processing and analysis}

In addition to the dataset generated in this study, raw reads generated by our previous study with bulls fed conventional diet (conventional group) were retrieved from the SRA database [accession number PRJNA525838], and processed to infer the impact of dietary treatments and to search for association with phenotypes.

Raw reads from conventional and byproduct groups were filtered for quality ( $>$ Q25) and trimmed at positions 220 (forward) and 175 (reverse) using QIIME 2 (version 2018.8)(Bolyen et al., 2019). These positions were selected based on aggregation plots provided by QIIME2. The filtered data was submitted to the DADA2 package to generate Amplicon Sequence Variants (ASVs) with the option just-concatenate, and exclude chimeric sequences (Callahan et al., 2016). Bacterial sequences were annotated using the 
SILVA database version 132 (Quast et al., 2013), archaeal sequences using the Rumen and Intestinal Methanogen database (RIM-DB) (Seedorf et al., 2014), and protozoa using a curated database containing protozoa 18S rRNA gene sequences (Kittelmann et al., 2015). The resulting ASV table was used to determine alpha (Number of ASVs and Shannon-Wiener index) and beta diversities (Unweighted Unifrac distance) with QIIME 2.

\section{Statistical analysis}

We contrasted the microbiome of groups submitted to different dietary treatments using the Analysis of Composition of Microbiomes (ANCOM) V2.1 (Kaul et al., 2017), with significance values adjusted for multiple tests using the Benjamin-Hochberg method $(\alpha<0.05)$. We applied a conservative W-statistic (Wstatistic cutoff $=0.9$ ), in which an ASV was considered as differential abundant if its composition varied when compared to $90 \%$ of the rest of the dataset, being the W-value the number of times the null hypothesis was rejected for a given ASV across two groups. ANCOM is a statistical approach that compares Aitchison's centered log-ratio transformed abundances of each ASV individually with all the remaining ASVs without any distributional assumptions (Kaul et al., 2017).

ASV abundances were tested for associations with animal phenotypes, such as $\mathrm{RCH}_{4}$ and RFI phenotypes using the Microbiome Multivariable Association with Linear Models (Maaslin) version 2 (Mallick et al., 2021), a multivariate model developed for microbiome data analysis, as it considers the compositional nature of these datasets. The analysis was adjusted for treatment, and contemporary groups using the following formula:

m0: CLR(ASV) Phenotype $\left(\mathrm{RFI}\right.$ or $\left.\mathrm{RCH}_{4}\right)+$ Diet + $\mathrm{CG}+$ residual error

Where CLR is the ASV abundance transformed using the centered log ratio method, and $\mathrm{e}$ is the residual error. ASVs identified as significant by the Maaslin analysis and that was prevalent in more than $20 \%$ of animals with a minimum abundance of $0.001 \%$, had their effect on the phenotypic variation (response variable) tested by using the linear mixed model implemented in Ime4qtl package (Ziyatdinov et al., 2018). Genetic relatedness was included as a random effect in the form of a kinship matrix to rule out the population structure effect in the phenotypic variation, jointly with diet and CG fitted as fixed effects. Genomic relatedness matrix was built in the AGHmatrix package (Amadeu et al., 2016) (Amadeu et al., 2016) of R (v. 4.0.3), based on the genomic matrix proposed elsewhere (VanRaden, 2008).

A likelihood ratio test was used to contrast models ( $\mathrm{m} 1$ and $\mathrm{m} 2$ ) to investigate the ASV impact on the phenotype. Our model can be described by the following formula: 
m1: phenotype $\left(\mathrm{RFI}\right.$ or $\left.\mathrm{RCH}_{4}\right) \sim$ Diet + CG + Kinship Matrix + residual error,

m2: phenotype( $\mathrm{RFI}$ or $\left.\mathrm{RCH}_{4}\right) \sim$ centered-log-ratio(ASV) + Diet + $\mathrm{CG}+$ Kinship Matrix + residual error.

Significant values for all models were adjusted for multiple tests using the false discovery rate (FDR) method $(a<=0.05)$.

\section{Results}

\section{Microbiome composition}

The sequencing of microbiome rRNA amplicons from ruminal and fecal samples of the byproduct group yielded a total of $10,573,763$ paired-end reads $(4,628,604$ paired-end reads for bacteria, $4,443,390$ for archaea, and 1,501,769 for protozoa), reaching 20,241,296 paired-end reads with the addition of sequencing data from the conventional group. After quality control, and singleton exclusion, a total of 4,519 bacterial ASVs (2,680 ruminal ASVs and 1,839 fecal ASVs), 1,023 archaeal ASVs (421 ruminal ASVs and 602 fecal ASVs) and 151 ruminal protozoa ASVs were identified across treatments.

Rarefaction curves based on the alpha-diversity metrics of Shannon-Wiener (diversity) reached a plateau, which indicated that additional sequences would not likely result in additional features.

Comparison of samples from different treatment groups using alpha-diversity metrics (Observed ASVs and Shannon-Wiener indexes) under the Kruskal-Wallis testing method revealed that rumen bacterial microbiome was significantly more abundant and richer in animals fed the conventional diet than those fed the by-products diet $(P=0.006$ and $P=0.04$, respectively). Similarly, the ruminal archaea diversity was also richer $(P=0.0004)$, but not more abundant. There was no significant difference when contrasting alpha diversity metrics of fecal samples. Comparisons of the beta-diversity metric Unweighted Unifrac using the PERMANOVA approach, revealed that samples of archaea and bacteria tended to form two significant clusters, which represented the treatment groups (adjusted $P<0.01$ ) (Supplementary Figures 1-3), a tendency most pronounced in fecal populations.

\section{Differential abundant ASVs in dietary treatment groups}

We applied the analysis of composition of microbiomes (ANCOM) to investigate the influence of dietary treatments in the microbiome composition at the ASV level. Seventeen ruminal ASVs of bacterial origin were differentially abundant (DA) with higher abundance in the conventional group, from which the most prominent were classified as Bacteroidales F082 group (ASV 20 and 23), Christensenellaceae (ASV 112), Pedosphaeraceae families (ASV 145) and the genus Succiniclasticum (ASV 170). Ten DA ASVs had 
higher abundance in the byproduct group, of which the most abundant were classified as Succiniclasticum (ASV 97), Acetitomaculum (ASV 116), Lachnospiraceae family (ASV 247), Fibrobacter (ASV 96) and Succinivibrio genus (ASV 118) (Supplementary Figure 4). Also, three fecal ASVs were DA in our experimental groups; one was classified as a member of the family Rikenellaceae (ASV 361) and was more abundant in the conventional group, while the ASV 332, classified as a member of the family Prevotellaceae, and ASV 526, classified as the genus Oscillibacter, () were both more abundant in the byproduct group (Supplementary Figure 5).

Eight archaeal ASVs were DA among treatment groups in the rumen microbiome. Four ASVs classified as M. gottschalkii (ASVs 1, 2, 13 and 11), one as M. ruminantium (ASV 23) and one ASV belonging to the Methanomassiliiicoccaceae family (ASV 36) were all more abundant in the conventional group, while one classified as M. ruminantium (ASV 4) and other as Methanosphaera group ISO3-F5 (ASV 33) were more abundant in the byproduct group (Supplementary Figure 6). Seven archaeal ASVs were DA in the fecal microbiome. From these, the ASVs classified as M. gottschalkii (ASVs 2, 13 and 11) and M. smithii (ASV 28) were more abundant in the conventional group, while M. ruminantium (ASV 4) and Methanosphaera group ISO3-F5 (ASVs 5 and 33) were more abundant in the byproduct group (Supplementary Figure 7). No ASVs of protozoa origin were observed as DA for any biome or group.

\section{Association between bacterial ASVs identified in the Nelore GIT and $\mathrm{CH}_{4}$ residual emission.}

We applied a generalized linear model (GLM) within the Maalin2 framework to investigate ASVs associated with $\mathrm{RCH}_{4}$ emission variation. This analysis allowed us to identify significant associations between bacteria and $\mathrm{RCH}_{4}$ in both environments. A second model was used to identify the phenotypic variation explained by significant ASVs identified by Maaslin2. We identified a single bacterial ASV associated with High- $\mathrm{RCH}_{4}$ (High emission) in the rumen, the ASV 3873 (coef_CLR=0.54, coef_RCH $\mathrm{RC}_{4}=10.8$, variation explained $=8.2 \%$ ), classified as a Solobacterium (Figure $1 \mathrm{~A}$ ), and one associated with Low- $\mathrm{RCH}_{4}$ (Low emission) in the stool environment, the ASV 85 (coef_CLR=-1.18, coef_RCH $4=-4.16$, variation explained=3.15\%), classified as Alistipes (Figure 1B). There were no significant associations between $\mathrm{RCH}_{4}$ emission and archaea or protozoa ASVs.

\section{Association between bacterial ASVs in the Nelore GIT and feed efficiency.}

Association analysis between RFI and microbiome components were performed using the same GLM and Mixed models described for the $\mathrm{RCH}_{4}$ analysis. We identified four bacterial ASVs associated with RFI in the rumen environment, three of these associated with feed inefficient $(\mathrm{H}-\mathrm{RFI})$ and one ASV associated with feed efficient (L-RFI) animals (Figure 2). We also identified one ASV associated with H-RFI and one with L-RFI in the stool environment (Figure 2). 
Among those associated with $\mathrm{H}-\mathrm{RFI}$, were the ASV 3718 (coef_CLR $=0.54$, coef_RFI= 0.72 , variation explained=9.53\%), classified as Christensenellaceae (gut group R-7), the ASV 1452 (coef_CLR $=0.71$, coef_RFI $=0.45$, variation explained $=5.96 \%$ ), classified as belonging to the Succinivibrio genus, ASV 813 (coef_CLR $=0.82$, coef_RFI=0.34, variation explained $=4.5 \%$ ), classified as belonging to the Prevotella genus in the rumen, and the ASV 4228 (coef_CLR $=0.49$, coef_RFI= 0.67 , variation explained $=8.87 \%$ ), classified as Ruminococcaceae UCG-005, in stool environments.

The ASV 1083 (coef_CLR = -0.91, coef_RFI=-0.39, variation explained=5.16\%) and ASV 260 (coef_CLR = -1, coef_RFI=-0.31, variation explained $=4.10 \%$ ), both classified as Rikenellaceae RC9, were significant in LRFI (Figure 2) in rumen and stool environments, respectively. There were no significant associations between RFI, and archaea or protozoa ASVs.

\section{Discussion}

In our previous study, we extensively explored the microbiome structure from two different sections of the Nelore cattle GIT (Andrade et al., 2020) under a single dietary treatment. Herein, we expand this study by introducing a new experimental group under a different diet and related the microbiome to production phenotypes. We compared these groups to investigate the impact of the diet on microbial abundance and diversity, as well as the contribution of individual ASVs on complex phenotypes, such as Residual Feed Intake (RFI) and Residual $\mathrm{CH}_{4}$ emission $\left(\mathrm{RCH}_{4}\right)$.

\section{The microbiome structure is affected by feed composition}

Analysis of alpha-diversity metrics showed that both bacteria and archaea only differed in the rumen environment, being less rich in animals of the byproduct group. This specific diet had citrus pectin in its composition, a polysaccharide that can greatly affect the microbiome composition (Larsen et al., 2019). Also, PCoA analysis of the Unweighted Unifrac beta-diversity metric highlights distinct clusters in treatment groups $A$ and $B$ for bacteria and archaea, but not for protozoa. Altogether, these results indicate that the diet is an important microbiome modulator, in agreement with previous studies in which the impact of different diets and feed components on microbiome diversity were evaluated (Dill-McFarland et al., 2019; Popova et al., 2019). However, such differences in the microbiome composition had little to no impact on the phenotypes studied herein, which can be partially explained by the microbiome functional redundancy described elsewhere (Tian et al., 2020).

Differential abundance analysis with bacterial ASVs revealed a significant impact of the dietary treatment in the bacterial populations of both environments. In the rumen environment, the ASVs classified as belonging to the Christensenellaceae family, as well as to the Prevotella and Fibrobacter genera, are known producers of Short-Chain Fatty Acids (SCFA), such as acetate, butyrate and propionate (Neumann et al., 2017; Waters and Ley, 2019), were identified as more abundant in the animals submitted to the conventional diet. While ASVs classified within genera known to produce succinate and propionate, i.e. Succiniclasticum and Succinivibrio (Hespell, 1992; van GYLSWYK, 1995), were identified as more 
abundant in the animals fed with the by-products diet. Differently from acetate and butyrate production, which increases $\mathrm{H}_{2}$ concentration in the rumen (Wolin, 1960), propionate is an electron acceptor endproduct of rumen fermentation and a viable alternative to methanogenesis (Ungerfeld, 2015; Wang et al., 2018). This process decreases methane energy loss and the greater amount of retained energy dilutes the maintenance costs, increasing efficiency. Another possible effect of higher propionate production would be DMI reduction (Allen et al., 2009), but that was not the case in this experiment.

The three DA ASVs identified in the fecal samples corresponded to bacteria that commonly inhabit the hindgut, such as the Oscillibacter genus and Prevotellaceae family, both more abundant in the byproduct group, and Rikenellaceae family, more abundant in the conventional group (Marounek and Duskova, 1999; Chen et al., 2011; Lee et al., 2012). The identification of a small number of DA ASVs in the fecal microbiome is consistent with the alpha diversity analysis, in which there was no significant difference in both abundance and richness among experimental groups.

The dietary treatment had also a significant impact on the archaea populations, e.g. an increased abundance of ASVs classified as M. gottschalkii in both rumen and stool environment of animals from conventional group, and $M$. ruminantium in both environments of animals from byproduct group. A study on sheep with contrasting phenotypes for $\mathrm{CH}_{4}$ emission found a higher abundance of the archaea $M$. gottschalkii in the higher emitter conventional group and M. ruminantium in the lower emitter group (Shi et al., 2014), although differences in $\mathrm{CH}_{4}$ emission were not observed between diets in our data.

\section{Phenotypic associations indicate biomarkers for $\mathrm{RCH}_{4}$ emission in the GIT microbiota}

GLM model analysis performed by the Maaslin V2 framework identified bacterial ASVs associated with the $\mathrm{RCH}_{4}$ phenotype in the rumen microbiome. An ASV classified as belonging to the Solobacterium genus was identified as the most significantly abundant bacterial ASV in animals with $\mathrm{H}-\mathrm{RCH}_{4}$ phenotype and explained $8.2 \%$ of the $\mathrm{RCH}_{4}$ variation. This genus was first described in 1999 (Kageyama and Benno, 2000) and has the species Solobacterium moorei as its sole representative. This bacterium is an important producer of volatile sulfur compounds (VSC) in the human oral microbiome (Stephen et al., 2014), such as hydrogen sulfide $\left(\mathrm{H}_{2} \mathrm{~S}\right)$, and this compound was previously identified in vitro as having inhibitory properties against methane oxidation (Lee et al., 2015), inhibiting methanotrophs from using $\mathrm{CH}_{4}$ as a carbon source.

In the stool microbiome, the ASV classified as belonging to the Alistipes genus was associated with L$\mathrm{RCH}_{4}$, explaining $3.1 \%$ of this phenotypic variation. This genus comprises gram-negative and anaerobic bacteria commonly identified in the bovine GIT microbiome(Dowd et al., 2008; Holman and Gzyl, 2019). Like other members of the Rikenellaceae family, bacteria from this genus produce acetate and propionate, both fatty acids with anti-inflammatory properties in the gut of humans and chickens(Polansky et al., 2015; Parker et al., 2020). Anyhow, the relationship between these taxa with methane emission and their biomarker/probiotic potential has to be further investigated using more layers of metagenomic information, such as metagenomes and metatranscriptomes. 
Surprisingly, we were not able to identify archaeal ASVs associated with $\mathrm{RCH}_{4}$ in both environments, although contributors to methane production, such as archaea belonging to the Methanobrevibacter genus, were highly abundant in the rumen microbiome, although the diet had a significant impact on the archaea population structure, as demonstrated by the DA analysis.

\section{Feed efficiency is linked to SCFA producers' in both environments.}

The ASVs significantly associated with H-RFI (inefficient animals) explained $20 \%$ of this phenotype variation in the rumen environment altogether were classified as Prevotella and Succinivibrio genus, and Christensenellaceae (gut group R-7). Species from the genus Prevotella have a major role in the digestion of complex polysaccharides, such as cellulose and hemicellulose, and have been identified as significantly more abundant in both spectra of feed efficiency in cattle (Carberry et al., 2012; HernandezSanabria et al., 2012; Myer et al., 2015), suggesting that the effect of Prevotella in the feed efficiency phenotype in cattle is species-specific (Perea et al., 2017).

Succinivibrio dextrinosolvens, the sole representative species of the Succinivibrio genus, presented an increased relative abundance in the rumen fluid of H-RFI Hereford $\times$ Aberdeen Angus steers (HernandezSanabria et al., 2012). This species is usually abundant in animals fed with high starch diets (O'Herrin and Kenealy, 1993) and produces formate and SCFA, such as acetate, succinate (Russell and Hespell, 1981). Formate can be reduced to $\mathrm{CO}_{2}, \mathrm{H}_{2}$, and $\mathrm{CH}_{4}$ by the action of rumen methanogens (Lovley et al., 1984), which can lead to a significant loss of feed energy and, consequently, a reduced feed efficiency. The Christensenellaceae (gut group R-7) produces acetate and butyrate as fermentation end products in the rumen and was identified as dominant in the ileum of high Feed Conversion Rate (FCR) pigs (Quan et al., 2018). This taxon was also identified as associated with methane emission in Holstein cows (Ramayo-Caldas et al., 2020). The only ASV associated with $\mathrm{H}$-RFI in the stool environment was classified as a Ruminococcaceae UCG-005. Members of this family were correlated with FCR of low efficient pigs (McCormack et al., 2017; Aliakbari et al., 2021).

\section{The potential of Rikenellaceae (gut group RC9) as biomarker for feed efficiency}

The ASVs associated with L-RFI (efficient animals) in both rumen and stool environments were taxonomically classified as belonging to the Rikenellaceae (gut group RC9). OTUs classified as belonging to this gut group were recently linked to low RFI in the rumen of Nelore steers, and to low FCR in the stool of pigs (Quan et al., 2018; Lopes et al., 2021), which, altogether, indicates a role of this specific taxon on this phenotype. The Rikenellaceae (gut group RC9), as other members of the Rikenellaceae family, can produce different SCFAs, such as propionate, acetate and succinate, some with anti-inflammatory properties (Tedelind et al., 2007; Parada Venegas et al., 2019). Gut microbes associated with antiinflammatory effects have been linked to gut health and with feed efficient pigs, as inflammation may redirect feed energy that, otherwise, could be used for host growth and weight gain (Gardiner et al., 2020), reinforcing their potential as a biomarker for highly efficient animals. 
The GIT is a continuous and interconnected system, but the knowledge regarding the stool microbiome in ruminants is limited when compared to the rumen microbiome for obvious reasons, however, it is a noninvasive sample that might represent a proxy for the rumen environment(Andrade et al., 2020). Nonetheless, the identification of biomarkers for phenotypes in stool is advantageous as its sampling is less stressful and can be implemented in the animal routine.

Studies and international consortia have been trying to mitigate $\mathrm{CH}_{4}$ emissions through the use of feed formulations, feed additives, and anti-methanogen vaccines(Moss et al., 2000). Some authors consider this problem intractable because the ruminal microbiota can rapidly adapt to external interventions (Roehe et al., 2016). However, there are promising results regarding early life treatments (Meale et al., 2021). Additional experiments have to be performed to test the potential markers identified in this study. However, understanding the biology of specific microorganisms that contribute to complex phenotypes may help to develop successful interventions for methane mitigation and feed efficiency in bovines.

\section{Conclusion}

The feed composition induced significant differences in abundance and richness of ruminal and fecal microbial populations. The dietary treatment based on industrial byproducts applied to our experimental groups influenced the microbiome diversity of bacteria and archaea, but not on protozoa. ASVs were associated with residual $\mathrm{CH}_{4}$ emission and residual feed intake in both ruminal and fecal microbiomes. While ruminal ASVs are expected to directly influence $\mathrm{CH}_{4}$ emission and feed efficiency, the relation of fecal taxa, such as Alistipes and Rikenellaceae (gut group RC9), with these traits might be associated with host health due to their link to anti-inflammatory compounds, and have the potential to be used as biomarkers for these complex phenotypes.

\section{List Of Abbreviations}

ASV - Amplicon Sequence Variant

CLR - Centered log-ratio

DA - Differentially abundant

FCR - Feed Conversion Rate

GIT - Gastrointestinal tract

$\mathrm{RCH}_{4}$ - Residual Methane Emission

RFI - Residual Feed Intake

SCFA - short-chain fatty-acid 


\section{Declarations}

\section{Ethics approval and consent to participate}

Experimental procedures were conducted following Brazilian guidelines on animal welfare and approved by the Ethics Committee on the Use of Animals, College of Veterinary and Animal Science, São Paulo State University under protocol $n^{\circ} 8510190118$.

\section{Availability of data and material}

All sequencing data are available in the NCBI Sequence Read Archive (SRA), under the bioproject number PRJNA638250.

\section{Competing interests}

All authors declare that the research was conducted in the absence of any commercial or financial relationships that could be construed as a potential conflict of interest

\section{Funding}

São Paulo Research Foundation (FAPESP - 2019/15557-7 and 2019/04089-2)

National Council for Scientific and Technological Development (CNPq - 4281153-6/2018)

\section{Acknowledgments}

We thank Dr. Marcela Morelli for collecting sample data, Dr. Leandro Sannomiya Sakamoto and the staff of the Embrapa Southeast Cattle Animal biotechnology laboratory for assistance on sample collection.

\section{Consent for publication}

Not applicable

\section{Authors contributions}

BGNA, JEK, AB, JCPP, JMR, LLC and LCAR conceived the experiment; BGNA and FAB performed the experiments; BGNA, RRCC, HA, JP and GBM performed analysis; BGNA, HA, RRCC, PSNO, AZN, JRM, SRM, MMS and LCAR interpreted the results; BGNA and LCAR drafted and revised the manuscript. All authors read and approved the final manuscript.

\section{Funding}

This research was conducted with funding from EMBRAPA, São Paulo Research Foundation scholarship to BGNA (FAPESP, grant number: 2019/15557-7), São Paulo Research Foundation (FAPESP, grant number: 2019/04089-2), the National Council for Scientific and Technological Development (CNPq, grant number: 428153/2018) and fellowships to LCAR and LLC. This research has received funding from the 
European Union's Horizon 2020 research and innovation programme under the Marie SkłodowskaCuriegrant agreement No. 801522, by Science Foundation Ireland and co-funded by the European Regional Development Fund through the ADAPT Centre for Digital Content Technology grant number 13/RC/2106.

\section{References}

Aliakbari, A., Zemb, O., Billon, Y., Barilly, C., Ahn, I., Riquet, J., et al. (2021). Genetic relationships between feed efficiency and gut microbiome in pig lines selected for residual feed intake. J Anim Breed Genet 138, 491-507. doi:10.1111/jbg.12539.

Allen, M. S., Bradford, B. J., and Oba, M. (2009). BOARD-INVITED REVIEW: The hepatic oxidation theory of the control of feed intake and its application to ruminants. Journal of Animal Science 87, 3317-3334. doi:10.2527/jas.2009-1779.

Amadeu, R. R., Cellon, C., Olmstead, J. W., Garcia, A. A. F., Resende, M. F. R., and Muñoz, P. R. (2016). AGHmatrix: R Package to Construct Relationship Matrices for Autotetraploid and Diploid Species: A Blueberry Example. Plant Genome 9. doi:10.3835/plantgenome2016.01.0009.

Andrade, B. G. N., Bressani, F. A., Cuadrat, R. R. C., Tizioto, P. C., Oliveira, P. S. N. D., Mourão, G. B., et al. (2020). The structure of microbial populations in Nelore GIT reveals inter-dependency of methanogens in feces and rumen. 1-10. doi:10.1186/s40104-019-0422-x.

Belkaid, Y., and Hand, T. W. (2014). Role of the Microbiota in Immunity and Inflammation. Cell 157, 121141. doi:10.1016/j.cell.2014.03.011.

Bolyen, E., Rideout, J. R., Dillon, M. R., Bokulich, N. A., Abnet, C. C., Al-Ghalith, G. A., et al. (2019). Reproducible, interactive, scalable and extensible microbiome data science using QIIME 2. Nature Biotechnology 37, 852-857. doi:10.1038/s41587-019-0209-9.

Callahan, B. J., McMurdie, P. J., Rosen, M. J., Han, A. W., Johnson, A. J. A., and Holmes, S. P. (2016). DADA2: High-resolution sample inference from Illumina amplicon data. Nature Methods 13, 581-583. doi:10.1038/nmeth.3869.

Carberry, C. A., Kenny, D. A., Han, S., McCabe, M. S., and Waters, S. M. (2012). Effect of phenotypic residual feed intake and dietary forage content on the rumen microbial community of beef cattle. Applied and environmental microbiology 78, 4949-58. doi:10.1128/AEM.07759-11.

Chang, J., Peng, S., Ciais, P., Saunois, M., Dangal, S., Herrero, M., et al. (2019). Revisiting enteric methane emissions from domestic ruminants and their $813 \mathrm{CCH} 4$ source signature reflecting diet and environmental shifts. doi:10.22022/ESM/06-2019.45. 
Chen, X., Zuo, Q., Hai, Y., and Sun, X. J. (2011). Lactulose: An indirect antioxidant ameliorating inflammatory bowel disease by increasing hydrogen production. Medical Hypotheses 76, 325-327. doi:10.1016/j.mehy.2010.09.026.

Cook, J., Oreskes, N., Doran, P. T., Anderegg, W. R. L., Verheggen, B., Maibach, E. W., et al. (2016). Consensus on consensus: A synthesis of consensus estimates on human-caused global warming. Environmental Research Letters 11. doi:10.1088/1748-9326/11/4/048002.

de Oliveira, M. N. V., Jewell, K. A., Freitas, F. S., Benjamin, L. A., Tótola, M. R., Borges, A. C., et al. (2013). Characterizing the microbiota across the gastrointestinal tract of a Brazilian Nelore steer. Veterinary microbiology 164, 307-14. doi:10.1016/j.vetmic.2013.02.013.

Dill-McFarland, K. A., Weimer, P. J., Breaker, J. D., and Suen, G. (2019). Diet Influences Early Microbiota Development in Dairy Calves without Long-Term Impacts on Milk Production. Applied and environmental microbiology 85, 1-12. doi:10.1128/AEM.02141-18.

Donoghue, K. A., Bird-Gardiner, T., Arthur, P. F., Herd, R. M., and Hegarty, R. S. (2016). Repeatability of methane emission measurements in Australian beef cattle. Anim. Prod. Sci. 56, 213. doi:10.1071/AN15573.

Dowd, S. E., Callaway, T. R., Wolcott, R. D., Sun, Y., McKeehan, T., Hagevoort, R. G., et al. (2008). Evaluation of the bacterial diversity in the feces of cattle using $16 \mathrm{~S}$ rDNA bacterial tag-encoded FLX amplicon pyrosequencing (bTEFAP). BMC microbiology 8, 125. doi:10.1186/1471-2180-8-125.

Flint, H. J., Duncan, S. H., Scott, K. P., and Louis, P. (2007). Interactions and competition within the microbial community of the human colon: links between diet and health. Environmental microbiology 9 , 1101-11. doi:10.1111/j.1462-2920.2007.01281.x.

Gardiner, G. E., Metzler-Zebeli, B. U., and Lawlor, P. G. (2020). Impact of Intestinal Microbiota on Growth and Feed Efficiency in Pigs: A Review. Microorganisms 8, 1886. doi:10.3390/microorganisms8121886.

Gilbert, J. a., and Dupont, C. L. (2011). Microbial Metagenomics: Beyond the Genome. Annual Review of Marine Science 3, 347-371.

Guan, L. L., Nkrumah, J. D., Basarab, J. A., and Moore, S. S. (2008). Linkage of microbial ecology to phenotype: correlation of rumen microbial ecology to cattle's feed efficiency. FEMS Microbiology Letters 288, 85-91. doi:10.1111/j.1574-6968.2008.01343.x.

Hernandez-Sanabria, E., Goonewardene, L. A., Wang, Z., Durunna, O. N., Moore, S. S., and Guan, L. L. (2012). Impact of Feed Efficiency and Diet on Adaptive Variations in the Bacterial Community in the Rumen Fluid of Cattle. Appl Environ Microbiol 78, 1203-1214. doi:10.1128/AEM.05114-11.

Hespell, R. B. (1992). "The Genera Succinivibrio and Succinimonas," in The Prokaryotes (New York, NY: Springer New York), 3979-3982. doi:10.1007/978-1-4757-2191-1_60. 
Holman, D. B., and Gzyl, K. E. (2019). A meta-analysis of the bovine gastrointestinal tract microbiota. FEMS Microbiology Ecology 95, fiz072. doi:10.1093/femsec/fiz072.

Kageyama, A., and Benno, Y. (2000). Phylogenic and Phenotypic Characterization of Some Eubacterium Like Isolates from Human Feces: Description of Solobacterium moorei Gen. Nov., Sp. Nov. Microbiology and Immunology 44, 223-227. doi:10.1111/j.1348-0421.2000.tb02487.x.

Kaul, A., Mandal, S., Davidov, O., and Peddada, S. D. (2017). Analysis of microbiome data in the presence of excess zeros. Frontiers in Microbiology 8, 1-10. doi:10.3389/fmicb.2017.02114.

Kittelmann, S., Devente, S. R., Kirk, M. R., Seedorf, H., Dehority, B. A., and Janssen, P. H. (2015). Phylogeny of Intestinal Ciliates, Including Charonina ventriculi, and Comparison of Microscopy and 18S rRNA Gene Pyrosequencing for Rumen Ciliate Community Structure Analysis. Applied and Environmental Microbiology 81, 2433-2444. doi:10.1128/aem.03697-14.

Koch, R. M., Swiger, L. A., Chambers, D., and Gregory, K. E. (1963). Efficiency of Feed Use in Beef Cattle. Journal of Animal Science 22, 486-494. doi:10.2527/jas1963.222486x.

Larsen, N., De Souza, C. B., Krych, L., Cahú, T. B., Wiese, M., Kot, W., et al. (2019). Potential of pectins to beneficially modulate the gut microbiota depends on their structural properties. Frontiers in Microbiology 10, 1-13. doi:10.3389/fmicb.2019.00223.

Lassen, J., and Difford, G. F. (2020). Review: Genetic and genomic selection as a methane mitigation strategy in dairy cattle. Animal 14, s473-s483. doi:10.1017/S1751731120001561.

Lee, E.-H., Moon, K.-E., Kim, T. G., Lee, S.-D., and Cho, K.-S. (2015). Inhibitory effects of sulfur compounds on methane oxidation by a methane-oxidizing consortium. Journal of Bioscience and Bioengineering 120, 670-676. doi:10.1016/j.jbiosc.2015.04.006.

Lee, G.-H., Kumar, S., Lee, J.-H., Chang, D.-H., Kim, D.-S., Choi, S.-H., et al. (2012). Genome Sequence of Oscillibacter ruminantium Strain GH1, Isolated from Rumen of Korean Native Cattle. Journal of Bacteriology 194, 6362-6362. doi:10.1128/JB.01677-12.

Lopes, D. R. G., de Souza Duarte, M., La Reau, A. J., Chaves, I. Z., de Oliveira Mendes, T. A., Detmann, E., et al. (2021). Assessing the relationship between the rumen microbiota and feed efficiency in Nellore steers. J Animal Sci Biotechnol 12, 79. doi:10.1186/s40104-021-00599-7.

Lovley, D. R., Greening, R. C., and Ferry, J. G. (1984). Rapidly growing rumen methanogenic organism that synthesizes coenzyme $M$ and has a high affinity for formate. Appl Environ Microbiol 48, 81-87. doi:10.1128/aem.48.1.81-87.1984.

Mallick, H., Rahnavard, A., Mclver, L. J., Ma, S., Zhang, Y., Tickle, T. L., et al. (2021). Multivariable Association Discovery in Population-scale Meta-omics Studies. bioRxiv, 2021.01.20.427420. 
Marounek, M., and Duskova, D. (1999). Metabolism of pectin in rumen bacteria Butyrivibrio fibrisolvens and Prevotella ruminicola. Letters in Applied Microbiology 29, 429-433. doi:10.1046/j.1472765X.1999.00671.x.

McCormack, U. M., Curião, T., Buzoianu, S. G., Prieto, M. L., Ryan, T., Varley, P., et al. (2017). Exploring a possible link between the intestinal microbiota and feed efficiency in pigs. Applied and Environmental Microbiology 83, 1-16. doi:10.1128/AEM.00380-17.

Meale, S. J., Popova, M., Saro, C., Martin, C., Bernard, A., Lagree, M., et al. (2021). Early life dietary intervention in dairy calves results in a long-term reduction in methane emissions. Sci Rep 11, 3003. doi:10.1038/s41598-021-82084-9.

Moss, A. R., Jouany, J. P., and Newbold, J. (2000). Methane production by ruminants: Its contribution to global warming. Animal Research 49, 231-253. doi:10.1051/animres:2000119.

Mudadu, M. A., Porto-Neto, L. R., Mokry, F. B., Tizioto, P. C., Oliveira, P. S. N., Tullio, R. R., et al. (2016). Genomic structure and marker-derived gene networks for growth and meat quality traits of Brazilian Nelore beef cattle. BMC Genomics 17, 235. doi:10.1186/s12864-016-2535-3.

Myer, P. R., Smith, T. P. L., Wells, J. E., Kuehn, L. A., and Freetly, H. C. (2015). Rumen microbiome from steers differing in feed efficiency. PLoS ONE 10, 1-17. doi:10.1371/journal.pone.0129174.

Neumann, A. P., McCormick, C. A., and Suen, G. (2017). Fibrobacter communities in the gastrointestinal tracts of diverse hindgut-fermenting herbivores are distinct from those of the rumen. Environmental microbiology 19, 3768-3783. doi:10.1111/1462-2920.13878.

Noel, S. J., Olijhoek, D. W., Mclean, F., Løvendahl, P., Lund, P., and Højberg, O. (2019). Rumen and Fecal Microbial Community Structure of Holstein and Jersey Dairy Cows as Affected by Breed, Diet, and Residual Feed Intake. Animals 9, 498. doi:10.3390/ani9080498.

O'Herrin, S. M., and Kenealy, W. R. (1993). Glucose and carbon dioxide metabolism by Succinivibrio dextrinosolvens. Applied and Environmental Microbiology 59, 748-755.

Pachauri, R. K., Mayer, L., and Intergovernmental Panel on Climate Change eds. (2015). Climate change 2014: synthesis report. Geneva, Switzerland: Intergovernmental Panel on Climate Change.

Parada Venegas, D., De la Fuente, M. K., Landskron, G., González, M. J., Quera, R., Dijkstra, G., et al. (2019). Short Chain Fatty Acids (SCFAs)-Mediated Gut Epithelial and Immune Regulation and Its Relevance for Inflammatory Bowel Diseases. Front. Immunol. 10, 277. doi:10.3389/fimmu.2019.00277.

Parker, B. J., Wearsch, P. A., Veloo, A. C. M., and Rodriguez-Palacios, A. (2020). The Genus Alistipes: Gut Bacteria With Emerging Implications to Inflammation, Cancer, and Mental Health. Front. Immunol. 11, 906. doi:10.3389/fimmu.2020.00906. 
Perea, K., Perz, K., Olivo, S. K., Williams, A., Lachman, M., Ishaq, S. L., et al. (2017). Feed efficiency phenotypes in lambs involve changes in ruminal, colonic, and small-intestine-located microbiota1. Journal of Animal Science 95, 2585-2592. doi:10.2527/jas.2016.1222.

Polansky, O., Sekelova, Z., Faldynova, M., Sebkova, A., Sisak, F., and Rychlik, I. (2015). Important Metabolic Pathways and Biological Processes Expressed by Chicken Cecal Microbiota. Appl Environ Microbiol 82, 1569-1576. doi:10.1128/AEM.03473-15.

Popova, M., Guyader, J., Silberberg, M., Seradj, A. R., Saro, C., Bernard, A., et al. (2019). Changes in the Rumen Microbiota of Cows in Response to Dietary Supplementation with Nitrate, Linseed, and Saponin Alone or in Combination. Applied and Environmental Microbiology 85, 1-16. doi:10.1128/AEM.02657-18.

Pszczola, M., Strabel, T., Mucha, S., and Sell-Kubiak, E. (2018). Genome-wide association identifies methane production level relation to genetic control of digestive tract development in dairy cows. Scientific reports 8, 15164. doi:10.1038/s41598-018-33327-9.

Quan, J., Cai, G., Ye, J., Yang, M., Ding, R., Wang, X., et al. (2018). A global comparison of the microbiome compositions of three gut locations in commercial pigs with extreme feed conversion ratios. Scientific Reports 8, 1-10. doi:10.1038/s41598-018-22692-0.

Quast, C., Pruesse, E., Yilmaz, P., Gerken, J., Schweer, T., Yarza, P., et al. (2013). The SILVA ribosomal RNA gene database project: Improved data processing and web-based tools. Nucleic Acids Research 41, 590596. doi:10.1093/nar/gks1219.

Ramayo-Caldas, Y., Zingaretti, L., Popova, M., Estellé, J., Bernard, A., Pons, N., et al. (2020). Identification of rumen microbial biomarkers linked to methane emission in Holstein dairy cows. Journal of Animal Breeding and Genetics 137, 49-59. doi:10.1111/jbg.12427.

Roehe, R., Dewhurst, R. J., Duthie, C. A., Rooke, J. A., McKain, N., Ross, D. W., et al. (2016). Bovine Host Genetic Variation Influences Rumen Microbial Methane Production with Best Selection Criterion for Low Methane Emitting and Efficiently Feed Converting Hosts Based on Metagenomic Gene Abundance. PLoS Genetics 12, 1-20. doi:10.1371/journal.pgen.1005846.

Russell, J. B., and Hespell, R. B. (1981). Microbial Rumen Fermentation. Journal of Dairy Science 64, 1153-1169. doi:10.3168/jds.S0022-0302(81)82694-X.

Sahoo, P. K., Kim, K., and Powell, M. A. (2016). Managing Groundwater Nitrate Contamination from Livestock Farms: Implication for Nitrate Management Guidelines. Curr Pollution Rep 2, 178-187. doi:10.1007/s40726-016-0033-5.

Seedorf, H., Kittelmann, S., Henderson, G., and Janssen, P. H. (2014). RIM-DB: a taxonomic framework for community structure analysis of methanogenic archaea from the rumen and other intestinal environments. PeerJ 2, e494. doi:10.7717/peerj.494. 
Shi, W., Moon, C. D., Leahy, S. C., Kang, D., Froula, J., Kittelmann, S., et al. (2014). Methane yield phenotypes linked to differential gene expression in the sheep rumen microbiome. 1517-1525. doi:10.1101/gr.168245.113.1.

Solden, L., Lloyd, K., and Wrighton, K. (2016). The bright side of microbial dark matter: lessons learned from the uncultivated majority. Current opinion in microbiology 31, 217-226.

doi:10.1016/j.mib.2016.04.020.

Tedelind, S., Westberg, F., Kjerrulf, M., and Vidal, A. (2007). Anti-inflammatory properties of the short-chain fatty acids acetate and propionate: A study with relevance to inflammatory bowel disease. WJG 13, 2826. doi:10.3748/wjg.v13.i20.2826.

Tian, L., Wang, X.-W., Wu, A.-K., Fan, Y., Friedman, J., Dahlin, A., et al. (2020). Deciphering functional redundancy in the human microbiome. Nat Commun 11, 6217. doi:10.1038/s41467-020-19940-1.

Ungerfeld, E. M. (2015). Shifts in metabolic hydrogen sinks in the methanogenesis-inhibited ruminal fermentation: a meta-analysis. Frontiers in Microbiology 6. doi:10.3389/fmicb.2015.00037.

van GYLSWYK, N. O. (1995). Succiniclasticum ruminis gen. nov., sp. nov., a Ruminal Bacterium Converting Succinate to Propionate as the Sole Energy-Yielding Mechanism. International Journal of Systematic Bacteriology 45, 297-300. doi:10.1099/00207713-45-2-297.

VanRaden, P. M. (2008). Efficient Methods to Compute Genomic Predictions. Journal of Dairy Science 91, 4414-4423. doi:10.3168/jds.2007-0980.

Wang, K., Nan, X., Chu, K., Tong, J., Yang, L., Zheng, S., et al. (2018). Shifts of hydrogen metabolism from methanogenesis to propionate production in response to Replacement of forage fiber with non-forage fiber sources in diets in vitro. Frontiers in Microbiology 9, 1-12. doi:10.3389/fmicb.2018.02764.

Waters, J. L., and Ley, R. E. (2019). The human gut bacteria Christensenellaceae are widespread, heritable, and associated with health. BMC Biology 17, 1-11. doi:10.1186/s12915-019-0699-4.

Williams, M., Zalasiewicz, J., Haff, P., Schwägerl, C., Barnosky, A. D., and Ellis, E. C. (2015). The Anthropocene biosphere. The Anthropocene Review 2, 196-219. doi:10.1177/2053019615591020.

Wolin, M. J. (1960). A Theoretical Rumen Fermentation Balance. Journal of Dairy Science 43, 14521459. doi:10.3168/jds.S0022-0302(60)90348-9.

Ziyatdinov, A., Vázquez-Santiago, M., Brunel, H., Martinez-Perez, A., Aschard, H., and Soria, J. M. (2018). Ime4qtl: linear mixed models with flexible covariance structure for genetic studies of related individuals. BMC Bioinformatics 19, 68. doi:10.1186/s12859-018-2057-x.

\section{Figures}




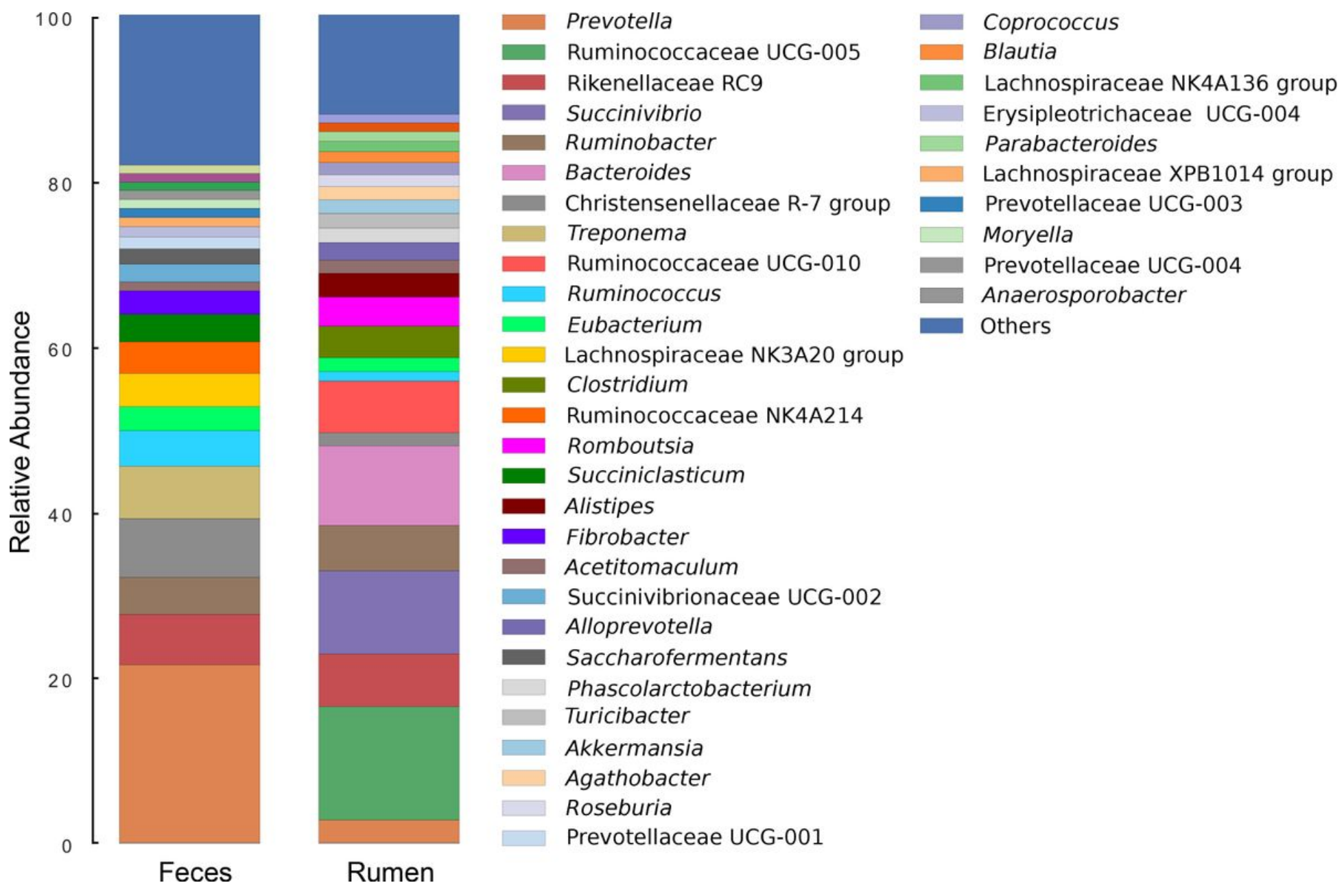

Figure 1

Variation of residual methane emission ( $\mathrm{RCH} 4$ ) versus the module abundance (CLR) variation of Amplicon Sequence Variants (ASV) in: A) Rumen; B) Stool. Both the phenotypic variation and ASV abundance variation were retrieved from the beta coefficients of Mixed-Models and Maaslin2 GLM regressions. Taxonomic information generated by Qiime2 was included. 
A)

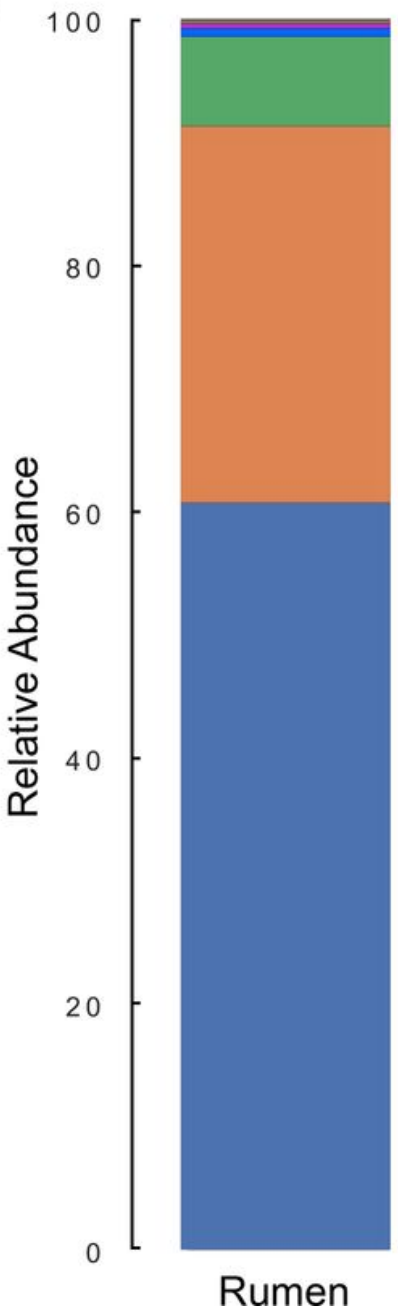

B)

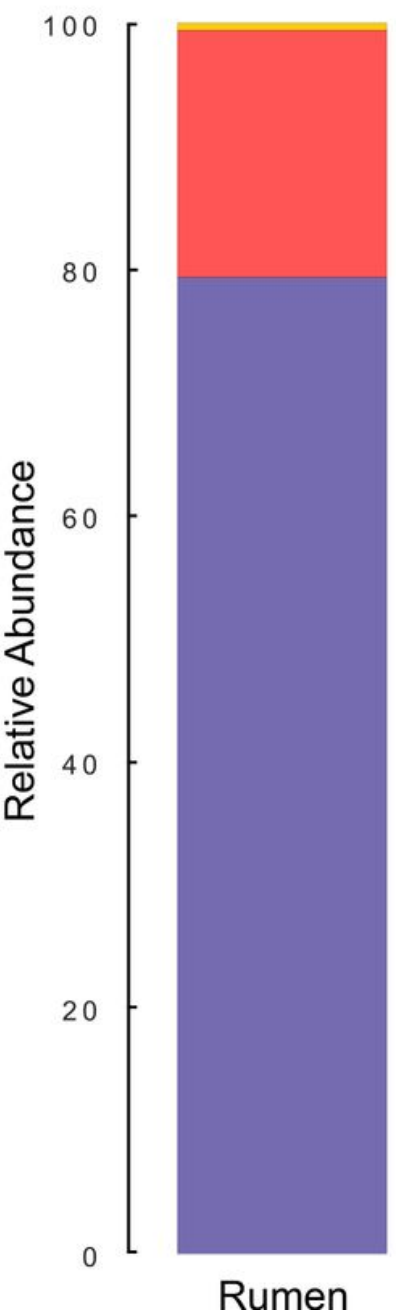

Methanobrevibacter gottschalkii Methanobrevibacter ruminantium Methanosphaera ISO3-F5 Methanobrevibacter smithii

Bozasella/Triplumaria

Entodinium

Ostracodinium

Figure 2

Variation of residual feed intake (RFI) versus the module abundance (CLR) variation of Amplicon Sequence Variants (ASV) in A) Rumen; B) Stool. Both the phenotypic and ASV abundance variations were retrieved from the beta coefficients of Mixed-Models and Maaslin2 GLM regressions. Taxonomic information generated by Qiime2 was included in the legend.

\section{Supplementary Files}

This is a list of supplementary files associated with this preprint. Click to download.

- SupplementaryData.docx

- SupplementaryFigure1.pdf

- SupplementaryFigure2.pdf

- SupplementaryFigure3.pdf

- SupplementaryFigure4.pdf

- SupplementaryFigure5.pdf 
- SupplementaryFigure6.pdf

- SupplementaryFigure7.pdf

Page 23/23 